ESTRATEGIAS DE MARKETING PARA LA GENERACIÓN DE CONFIANZA EN LAS COMPRAS POR INTERNET

\title{
ESTRATEGIAS DE MARKETING PARA LA GENERACIÓN DE CONFIANZA EN LAS COMPRAS POR INTERNET
}

\section{MARKETING STRATEGIES FOR THE GENERATION OF CONFIDENCE ON \\ ONLINE SHOPPING}

Gabriela del Carmen Díaz Vázquez*

*Licenciada en Informática Administrativa. Maestra en Administración. Universidad Juárez Autónoma de Tabasco.

Dirección para recibir correspondencia: gabrielladiaz.v@gmail.com 
ESTRATEGIAS DE MARKETING PARA LA GENERACIÓN DE CONFIANZA EN LAS COMPRAS POR INTERNET

\section{RESUMEN}

OBJETIVO: Identificar factores generadores de confianza en las compras por internet en los jóvenes del municipio de Centro, Tabasco; estado situado en la zona sureste de México, zona con menor porcentaje de población conectada a internet.

MATERIAL Y MÉTODO: Investigación de tipo no experimental con enfoque mixto. Los hallazgos se obtuvieron a través de una encuesta.

RESULTADOS: Se identifica la existencia de factores cognitivos importantes que permiten que se genere en el consumidor una actitud de aceptación y de confianza hacia internet como canal de compra más allá del factor tecnológico, el cual es mayormente asociado al hablar de seguridad en internet y que hace referencia únicamente a la infraestructura tecnológica empleada en el desarrollo del Sitio Web. A través de estos factores se logra la identificación de estrategias de marketing aplicables para la aceptación de la compra por internet en los jóvenes tabasqueños.

CONCLUSIONES: El comercio electrónico tiene presencia en el consumidor actual, sabe de su existencia y beneficios, se informa a través de páginas oficiales de marcas o tiendas en línea sobre los productos que le interesan, solo necesita estímulos para concretar la compra y aumentar su acercamiento y aceptación.

Palabras clave: Comercio electrónico. Confianza. Consumidor. Estrategias. Marketing. 
ESTRATEGIAS DE MARKETING PARA LA GENERACIÓN DE CONFIANZA EN LAS COMPRAS POR INTERNET

\section{ABSTRACT}

OBJECTIVE: Identifying factors that generate confidence on online shopping done by young people of the municipality of Centro, Tabasco; state located in the southeast region of the Mexican Republic, area with a lower percentage of population connected to the internet.

MATERIAL AND METHOD: Research of non-experimental type with mixed approach. The findings were obtained through a survey.

RESULTS: It is identified the existence of important cognitive factors that generate in the consumer an acceptance and trust attitude towards internet as a purchasing channel beyond the technological factor, which is mostly associated when referring to Internet security and that refers solely to the technological infrastructure used in the development of the website. Through these factors it can be reached the identification of marketing strategies applicable to the acceptance of online purchasing among young people from Tabasco.

CONCLUSIONS: E-commerce has a presence in today's consumer, who knows about its existence and benefits, it is informed through official brands and online stores pages about the products of their interest, the only thing that they need is a series of stimuli to concrete the purchase and increase its approach and acceptance.

KEYWORDS: E-commerce. Confidence. Consumer. Strategies. Marketing. 
ESTRATEGIAS DE MARKETING PARA LA GENERACIÓN DE CONFIANZA EN LAS COMPRAS POR INTERNET

\section{INTRODUCCIÓN}

En un entorno virtual, el grado de incertidumbre de las transacciones económicas es mayor que en los entornos tradicionales ya que pueden ser susceptibles de varios riesgos, como los derivados de la infraestructura tecnológica o por la conducta de los actores que participan. Por esta razón, las actitudes hacia las compras por internet se ven afectadas por las perturbaciones de los consumidores al momento de realizar una adquisición en este medio.

Por otro lado, el éxito en un modelo de negocio electrónico depende de la estrategia de marketing. La confianza, por su parte, es un aspecto estratégico y elemento clave, ya que conseguir ventas no es lo principal, sino construir una relación sólida con el usuario hasta que esté preparado para comprar. Tomando como base lo anterior, se determina el importante papel del marketing y sus estrategias para fomentar confianza en el consumidor hacia el comercio electrónico y posterior crecimiento de más negocios y emprendedores que exploten los beneficios de este canal.

\section{Antecedentes}

En México, el número de usuarios de internet ha crecido así como la aceptación por la compra en internet, pero no a la par. "En el 2015, la población conectada a Internet llego a 65 millones de personas, alcanzando el 59\% de penetración, de los cuales la mayoría son jóvenes entre 13-34 años de edad". En cuanto al tema del comercio electrónico, "38\% de la población conectada a internet realiza compras en línea", de lo anterior, resulta importante destacar que "del total de compras online realizadas en México, más de la mitad se compró fuera del país; esto con un total de 60\%, siendo Estados Unidos el principal país con 64\%" (AMIPCI, 2016).

De igual forma, en México, los estados en sur y sureste son los que contemplan el menor porcentaje de población conectada a internet con un $6 \%$ y $7 \%$ respectivamente, a comparación de estados con una mayor afluencia como: Centro 26\% y Noroeste 15\% (AMIPCI, 2016). Por lo cual, si no existe una mayor familiaridad con el entorno digital por parte de los individuos, estos difícilmente llevarían a cabo una compra por este medio.

Tabasco es un estado situado en el sureste de México, cuenta con 17 municipios donde la ciudad de Villahermosa es capital del estado y se localiza en el municipio de Centro, cuenta con una población total de jóvenes entre 18 a 34 años de edad de 170,512; de acuerdo con estudios los jóvenes en este rango de edad son los principales empleadores del comercio electrónico. 
ESTRATEGIAS DE MARKETING PARA LA GENERACIÓN DE CONFIANZA EN LAS COMPRAS POR INTERNET

La presente investigación tiene por objetivo la identificación de factores generadores de confianza en internet como canal de compra, identificados mediante una revisión y análisis literario, de igual forma resulta de importancia validar y valorizar esta información a través de la opinión de una población que se considera no estar tan inmersa en el entorno online, debido a que el tema central se enfoca en la generación de confianza. Por ello, en esta información se identifican estrategias de marketing que fomenten la aceptación de la compra por internet y permitan un mayor crecimiento del comercio electrónico, no solo en el estado, sino en el país y generar oportunidades de crecimiento económico con el desarrollo de negocios de venta online a través de jóvenes emprendedores que fijen su mira en este ámbito, reconociendo las oportunidades que internet les ofrece, e incluso para aquellos negocios físicos que quieran expandirse y llevar sus productos y servicios a otros lugares.

\section{Hipótesis}

Generando los factores que contribuyen a la confianza en internet como canal de compra y aplicando estrategias adecuadas de marketing, enfocadas en estos, se puede lograr la aceptación del consumidor por el comercio electrónico.

\section{Marco teórico}

\section{Conceptos de comercio electrónico}

La OECD (1997) define al comercio electrónico como una transacción comercial sobre redes abiertas como internet. Se incluyen tanto las transacciones de empresa a empresa como las de empresa a consumidor.

Para Boen (2004) el comercio electrónico "constituye el intercambio de bienes, servicios e información por medio de los sitios electrónicos".

\section{Tipos de comercio electrónico}

Arango (2013) expone la siguiente clasificación de las modalidades del comercio electrónico:

(B2B, Business to Business) Negocio a negocio. Las actividades comerciales son entre empresas y no con el usuario final, las actividades están ligadas con fabricantes, distribuidores y comercio minorista. 
ESTRATEGIAS DE MARKETING PARA LA GENERACIÓN DE CONFIANZA EN LAS COMPRAS POR INTERNET

(B2C, Business to Consumer) Negocio a consumidor. Las actividades comerciales son entre una empresa y consumidor, el consumidor hace uso de la página buscando productos de su interés, si encuentra lo que necesita realiza un pago por ello. Trata de portales que ofrecen productos servicios y contenidos a personas físicas, en las fases de preventa, venta y postventa.

(B2A, Business to Administration) Entre empresas y la administración. Forman parte de la comunicación global de la compañía como pagos de impuestos. Es un servicio que ofrece la administración a las empresas y también a los ciudadanos para que se puedan realizar los trámites administrativos a través de Internet.

(C2C, Consumer to Consumer) Entre consumidores. Es un modelo de negocio B2C Facilita la comercialización de bienes y/o servicios entre particulares.

Después de haber definido cada una de las modalidades del comercio electrónico, cabe destacar que la investigación solo se enfoca al tipo Business to Consumer (B2C) o "Negocio a consumidor".

\section{Riesgos en internet y transacciones electrónicas}

Mafe \& Rios (2007) definen el riesgo percibido de compra como "la expectativa de pérdida del internauta cuando realiza una transacción electrónica determinada". Éste constituye una barrera que puede impedir que el consumidor decida optar por este canal. Los usuarios presentan mayor disponibilidad para hacer una transacción en línea cuando el riesgo que perciben es menor. En el comercio electrónico, el riesgo está aún más presente ya que la falta de tangibilidad del producto y del vendedor es un factor clave que crea una percepción de riesgo para el posible cliente.

García \& Álvarez (2000) afirman que en el mundo anónimo del internet, los clientes y los vendedores, deben enfrentarse a un conjunto de amenazas como son:

- Accesos no autorizados: Alguien hace un mal uso de un sistema de cómputo para interceptar información y robar datos delicados.

- Alteración de datos: En el contenido de una transacción comercial se modifican datos como: nombre, número de tarjeta de crédito, monto de la transacción, y estos son alterados durante su transmisión. 
ESTRATEGIAS DE MARKETING PARA LA GENERACIÓN DE CONFIANZA EN LAS COMPRAS POR INTERNET

- Monitoreo por personas ajenas: Se da al romper mecanismos de seguridad en las redes de computadoras (sin autorización) y monitorear la información confidencial que está siendo transmitida.

De acuerdo a la CONDUSEF (2016) la clonación es uno de los principales fraudes que sufren los usuarios de servicios financieros. Las posibles causas de presuntos fraudes en tarjetas son:

- Robo o extravío del plástico.

- Clonación de la banda magnética en cajeros o terminales.

- Compras por teléfono o por internet.

- Transferencias electrónicas.

- $\quad$ Falsificación de datos personales.

\section{Confianza en línea como medio de compra}

De acuerdo con Rojas, Arango, \& Galleo (2009) la confianza en línea se examina desde tres perspectivas:

1. Integridad (creencia de que el comerciante en línea se adhiriera a las reglas establecidas o mantenga lo prometido).

2. Capacidad (creencia acerca de las habilidades y las competencias del comerciante en línea para ofrecer productos y servicios de buena calidad).

3. Benevolencia (creencia de que el comerciante en línea, además de querer lograr beneficios legítimos, quiera hacer el bien al cliente sin referirse expresamente a hacer una venta).

Los autores proponen tres dimensiones de confianza:

1. La habilidad del comerciante en línea para entregar un producto o servicio que prometió.

2. La voluntad del comerciante en línea para rectificarle al cliente en caso de que la compra que éste realizó no cumpla con sus expectativas.

3. Presencia de una política o una declaración de privacidad sobre el sitio Web. 
ESTRATEGIAS DE MARKETING PARA LA GENERACIÓN DE CONFIANZA EN LAS COMPRAS POR INTERNET

\section{Modelo Teórico}

A partir de un proceso de revisión bibliográfica se determinan factores generadores de confianza en el comercio electrónico, lo anterior, a través de detectar similitudes entre diversos modelos de confianza en línea. Estos factores se muestran en la siguiente ilustración:

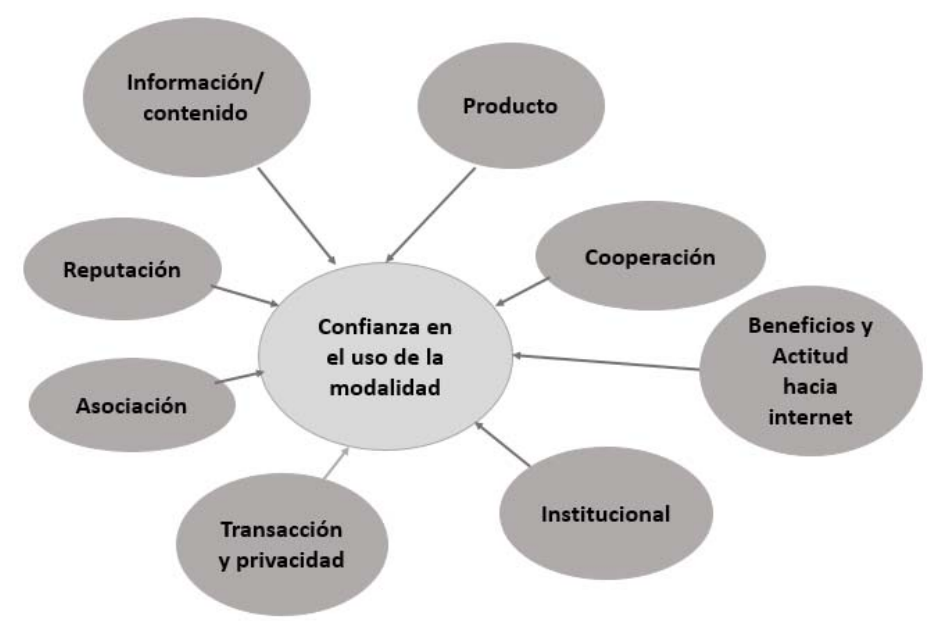

Figura 1. Factores generadores de confianza en la compra por internet.

Fuente: Elaboración propia.

- Información/Contenido: La percepción de facilidad de uso tiene una influencia positiva en la actitud de los consumidores en el uso de internet para comprar; permitir a estos comprar de la forma que quieran y con resultados fáciles resulta de importancia al igual que la creatividad, no solo en el diseño del portal sino en las imágenes, videos o información mostrados (contenido).

- Reputación: Hace referencia al comportamiento del vendedor o negocio, es decir, la imagen que se tiene de éste en base a opiniones positivas o negativas por parte de terceros.

- Asociación: Hace referencia a como una persona (A) relaciona la confianza con "algo" (B) que puede ser cualquier cosa: un nombre, persona, el precio de un producto o una opinión, y este "algo" es lo que le genera confianza en un tercer elemento (C). El proceso más básico por el que la confianza se construye es a partir de la asociación, confiamos en alguien o algo porque otros confían. Si el vendedor genera confianza en el entorno de referencia del comprador potencial, éste tendrá opiniones positivas sobre dicho vendedor, 
ESTRATEGIAS DE MARKETING PARA LA GENERACIÓN DE CONFIANZA EN LAS COMPRAS POR INTERNET

lo que puede incrementar la motivación del cliente para comportarse de manera similar a su entorno en las transacciones en línea.

- Transacción y privacidad: Hace referencia a diversos atributos que hacen transacciones en línea confiables como: transparencia, precios y pagos, políticas de reembolso, promociones, garantías. La privacidad es un elemento clave ya que tiene que ver con el respeto y mantenimiento de la confidencialidad de la información personal de quien compra.

- Institucional: Al igual que con la asociación y la reputación el factor institucional tiene que ver con "terceros" que contribuyen a la confianza en la compra por internet. Para este caso se refiere a la acreditación y aprobaciones legales que el portal es seguro para efectuar transacciones en internet.

- Beneficios y Actitud hacia internet: Hace referencia a crear en la mente del consumidor una actitud positiva hacia la compra por internet, tomando como base los beneficios que obtendrá en este canal a diferencia de una compra tradicional en tienda física. La actitud se considera como la evaluación u opinión general de las personas hacia algo.

- Cooperación: refleja la facilidad de interacción entre vendedor-comprador en la fundación de la confianza.

- Producto: Atributos de un producto que promueven o disuaden el intercambio en línea: durabilidad, valor de marco, fiabilidad, calidad personalización, disponibilidad.

\section{METODOLOGÍA}

\section{Diseño de la investigación y enfoque}

El diseño de la investigación es de tipo no experimental con un enfoque mixto, ya que contiene aspectos cualitativos representados por medio del análisis literario y aspectos cuantitativos en la medición y análisis de datos numéricos obtenidos mediante una encuesta.

\section{Población y muestra}

La población de estudio se conformó por jóvenes en edad de 18 a 34 años, ya que de acuerdo con estudios de la Asociación Mexicana de Internet (AMIPCI, 2016), el mayor número de compradores en línea mexicanos oscila en este rango de edad. La base de datos utilizada para la cuantificación de la información se obtuvo del Instituto Nacional de Estadística y Geografía 
ESTRATEGIAS DE MARKETING PARA LA GENERACIÓN DE CONFIANZA EN LAS COMPRAS POR INTERNET

(INEGI, 2010). De la cual se desprende una población total de 170,512 jóvenes entre 18 a 34 años de edad en el municipio de Centro, Tabasco.

Considerando el alto número de población se determina la conveniencia de realizar el cálculo de una muestra para estudiar la población. La muestra se calculó de la siguiente forma:

$$
\mathrm{N}=\frac{\mathrm{N} Z^{2} \text { p q }}{(\mathrm{N}-1) \mathrm{e}^{2}+\mathrm{Z}^{2} \mathrm{pq}}
$$

Donde:

$\mathrm{N}=$ Población total

$Z=$ Nivel de confianza. En este caso representa el 95\% $=1.96$

p: proporción de individuos que poseen en la población la característica de estudio. Este dato es desconocido y se suele suponer que $p=q=0.5$ que es la opción más segura.

q: proporción de individuos que no poseen esa característica, es decir, es 1-p. Al igual que "p" tendrá un valor de 0.5

$\mathrm{e}=$ Margen de error. En este caso representa 5\%

\section{Despeje de la fórmula:}

$$
\begin{aligned}
& N=\frac{170,512(1.96)^{2}(0.5)(0.5)}{(170,512-1)(0.05)^{2}+(1.96)^{2}(0.5)(0.5)} \\
& N=\frac{16375.7248}{427.2379} \\
& N=383.2986
\end{aligned}
$$

Como resultado del cálculo se obtuvo un total de 383, lo cual representa el número de personas a quienes se les aplicó el cuestionario. El tipo de muestreo empleado para la elección de los participantes fue el muestreo probabilístico. (Sampieri, Collado, \& Lucio, 2010) definen a esté como el "subgrupo de la población en el que todos los elementos de está tienen la misma posibilidad de ser elegidos".

\section{Técnicas e instrumentos}

El instrumento utilizado para la recolección de datos fue el cuestionario, toda vez que se adapta a esta investigación y consta de dos bloques para una mejor organización de la información. 
ESTRATEGIAS DE MARKETING PARA LA GENERACIÓN DE CONFIANZA EN LAS COMPRAS POR INTERNET

En el primer bloque se hacen preguntas para la identificación de la muestra tales como: sexo, edad y si el encuestado ha comprado alguna vez por internet; en el segundo bloque se indaga por cada uno de los factores que contribuyen a la generación de confianza en internet como canal de compra, los cuales fueron identificados en la revisión bibliográfica (ver figura 1). Este bloque fue construido con un total de 24 afirmaciones, distribuidas entre cada uno de los factores anteriores y medidas con una escala Likert de 1 a 5 puntos que comprende desde "Muy de acuerdo" hasta "Totalmente en desacuerdo".

Este método se adapta a la investigación, ya que nos permite medir actitudes y conocer el grado de conformidad del encuestado, resultando útil emplearla en situaciones en las que se desea que la persona matice su opinión.

\section{Validación de los instrumentos}

Para la construcción del instrumento de medición se tomó como referencia el trabajo de (Alzate, 2015), mismo que fue adaptado al contexto. La finalidad del instrumento es validar y valorizar los factores que contribuyen a la generación de confianza en internet como canal de compra y con los resultados obtenidos identificar estrategias de marketing enfocadas en generar aceptación hacia el comercio electrónico a través de la confianza.

\section{RESULTADOS}

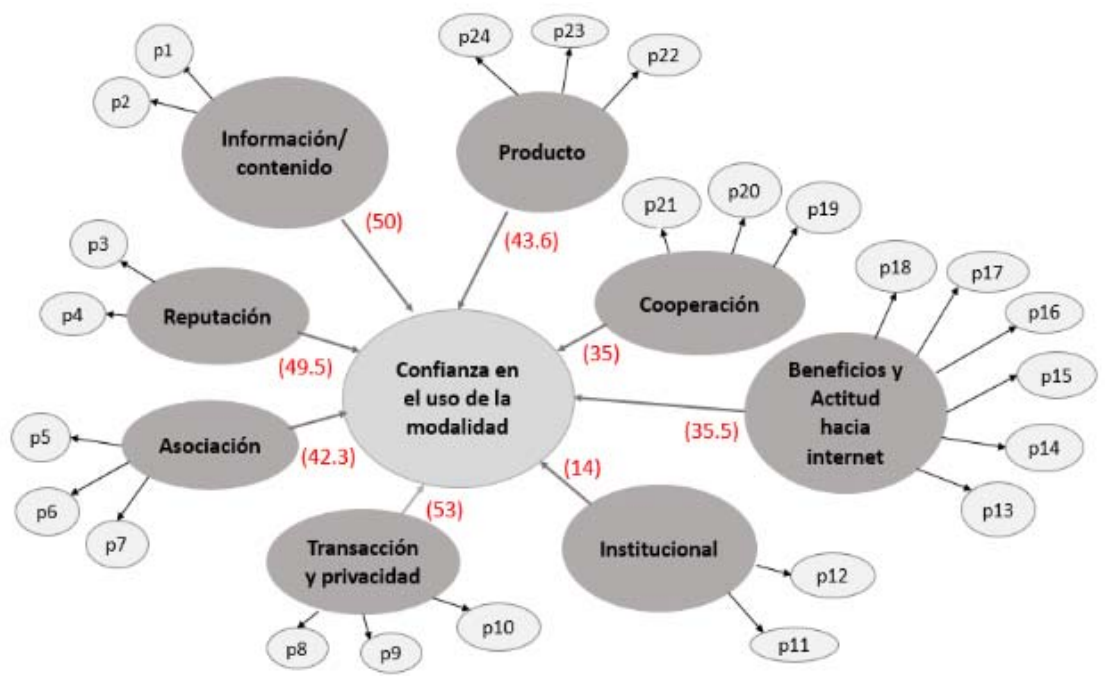

Figura 2. Resultados del modelo teórico.

Fuente: Elaboración propia. 
ESTRATEGIAS DE MARKETING PARA LA GENERACIÓN DE CONFIANZA EN LAS COMPRAS POR INTERNET

A traves del analisis de los resultados obtenidos en la encuesta, se determina el valor de importancia que tiene para los jovenes del municipio de Centro los factores que generan confianza en el comercio electronico (ver figura 2). Estos valores se obtienen en base a las respuestas con mayor porcentaje obtenido en cada afirmacion a traves de la escala Likert.

1. Transacción y privacidad.- es el principal factor generador de confianza para el consumidor, ya que implica aspectos de proteccion a la información personal y que tanto de esta estan dispuestos a proporcionar, los riesgos y garantias que el negocio/vendedor le ofrece al comprador y la entrega del producto.

2. Información/contenido.- al consumidor le genera confianza la apariencia del portal del negocio/vendedor, reconoce que la primera impresión cuenta y define a quien esta detrás. Buscan que sea: facil de interactuar, con información relevante y sin saturacion de contenido, atractivo a la vista, creativo.

3. Reputación.- los encuestados aceptan que se sienten/sentirian confiados al comprar por internet a una tienda de marca reconocida por la reputacion que esta ya se ha hecho en el mercado, pero de igual forma a través de comentarios positivos de terceros sobre sus experiencias actuarían de la misma forma con cualquier otro negocio/vendedor.

4. Producto.- es la motivación del consumidor a realizar una compra, es lo que desea adquirir, por lo cual estaría dispuesto a adentrarse en este canal si sabe que no tiene otra alternativa para adquirirlo.

5. Asociación.- la confianza de un individuo puede ser derivada de la combinacion de referencias recibidas que pueden ser personas cercanas, opiniones, precio, todo aquello que pueda interpretarse como una señal de "seguridad.

6. Beneficios y actitud hacia internet.- este factor decae en importancia debido a que en la actualidad se tiene una mayor interacción con las tecnologias e internet que en años anteriores. Los jovenes tienen una opinión positiva hacia este canal y reconocen las ventajas que proporciona en cuanto a adquirir productos extranjeros y con comodidad.

7. Cooperación.- la interacción con el vendedor es importante para el consumidor, sin embargo, como no existe un vinculo fisico con este, como en una compra tradicional, su relación se limita unicamente a la aclaración de dudas. Por lo cual decae en valor. 
ESTRATEGIAS DE MARKETING PARA LA GENERACIÓN DE CONFIANZA EN LAS COMPRAS POR INTERNET

8. Institucional.- obtiene el ultimo grado en importancia debido a que el consumidor no tiene un conocimiento claro de acreditaciones y sellos de garantía que catalogan a un portal como "seguro" para el comercio electrónico. De la misma forma, son neutrales en cuanto a su conocimiento de las instituciones que vigilan y protegen al consumidor en el comercio electrónico. Por lo cual, si no tienen un conocimiento claro de esto, desconocen que tan importante o no es para ellos.

\section{Propuesta: estrategias de marketing para la generación de confianza en las compras por internet.}

Derivado de la validación de los factores que generan confianza en internet como canal de compra se confirma en su totalidad su influencia en el mercado de estudio; aunado a estos se anexaron al cuestionario teorías personales y aspectos que eran necesarios conocer en función de aportación personal. De lo anterior, se proponen las siguientes estrategias de marketing para aumentar el crecimiento de la aceptación de los consumidores por el comercio electrónico.

\section{Las políticas del negocio deben estar visibles en el Portal.}

Es necesario que se cuenten con políticas sobre privacidad, reembolso en caso de tener un problema con el producto recibido (cambio o devolución del dinero) y garantías. Si se busca que el consumidor elija internet como canal de compra, se debe considerar ofrecerles los mismos o más beneficios y seguros que una tienda física haría. Se recomienda:

*Anexar un apartado para "Preguntas y respuestas" en el Portal con las dudas principales que se consideran tendrán los consumidores del canal de internet: tiempo de entrega, posibilidad de programarse un pedido, que pasa si no hay nadie para recibirlo, formas de pagos utilizables o seguridad de la información. Por ejemplo, sobre la entrega del producto, algunas empresas establecen tratos con paquetería donde estas se comprometen a hacer 3 intentos de entrega. En caso de no poder realizarla, por situaciones como el hecho de no haber nadie en el domicilio para recibirlo, el paquete se iría a la sucursal de la paquetería para que el cliente pase a recogerlo. Este tipo de información debe ser visible al consumidor.

\section{Monitoreo de cargos a tarjetas bancarias y paquetería.}

Actualmente los bancos ofrecen servicios de notificaciones vía mensajes (SMS) al celular, a través del cual el consumidor tiene conocimiento de los retiros o pagos que se hagan a sus tarjetas. Existen también aplicaciones móviles de empresas de paquetería que permiten al 
ESTRATEGIAS DE MARKETING PARA LA GENERACIÓN DE CONFIANZA EN LAS COMPRAS POR INTERNET

comprador monitorear el trayecto de su paquete. Esto permite al consumidor tener conocimiento de algún fraude y actuar en el momento. Dar a conocer esta información resulta de importancia en la labor de generar confianza ya que la tecnología está brindando importantes beneficios que muchas veces pasan desapercibidos.

\section{Evitar solicitar más información de la necesaria para la transacción y explicar la razón de ser de los datos que se solicitan.}

Nombre y primer apellido (Gabriela Díaz)

Dirección (entrega del paquete)

Ciudad/estado (entrega del paquete)

Número de tarjeta (pago)

Dirección (entrega del paquete), esta breve explicación disminuirá el sentimiento de riesgo o desconfianza al permitir que el comprador entienda la finalidad del dato durante la transacción. Lo anterior puede detallarse en la sección de "preguntas y respuestas" del Sitio Web, en un apartado de "proceso de compra" o anexarse en el formulario como un mensaje explicativo (Tooltip), el cual se verá automáticamente cuando el usuario pase el cursor sobre el recuadro que se requiere llenar con algún dato, esto puede hacerse durante el proceso de programación del Portal.

\section{Domicilio:}

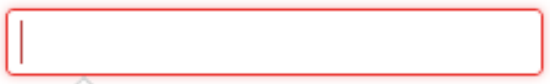

Sin este dato no podternos

errviar tu paquete

Figura 3. Tooltip.

Fuente: Elaboración propia. 
ESTRATEGIAS DE MARKETING PARA LA GENERACIÓN DE CONFIANZA EN LAS COMPRAS POR INTERNET

\section{Generar una opinión confiable de la marca o vendedor a través del diseño del portal.}

Como en un local o tienda física se diseña cada aspecto interno de la tienda para generar una imagen de la marca y atraer al consumidor, de igual forma se debe considerar al portal, al ser lo que ven las personas al entrar a este y que refleja al negocio/vendedor creando una atmosfera de confianza, credibilidad y seguridad. Es importante invertir el tiempo, la dedicación y recursos en:

- Diseñar en función de la Web responsiva, es decir, pensando en que el diseño se ajuste a la pantalla de cualquier dispositivo, sin modificar su contenido. La compra por internet ya no se realiza exclusivamente por computadora.

- Tomar a consideración las sugerencias de teorías de color aplicados al diseño Web actuales, contenido, distribución de la información.

- Recordar que las nuevas generaciones (Millenials y Generación Z) son quienes más interactúan en internet, por lo cual si buscamos captar su interés debemos ser creativos y estar al tanto de sus gustos y preferencias que puedan reflejarse en nuestro contenido y diseño para que se sientan familiarizados.

\section{La asociación es clave en la generación de confianza en las compras por internet.}

a) Asociar el comercio electrónico con la reputación. Para que un consumidor acepte la compra por internet los comentarios positivos son importantes, con estos puede llegar a percibir tan confiable y seguro al comercio electrónico y a un Portal, como aquellos con una marca consolidada o extranjeros. Para esto se recomienda:

*Uso de medios sociales: a través de ellos se emiten opiniones de experiencia. Se tiene que dejar de ver y emplear a estos como una herramienta exclusiva de publicidad, si no para conectar con el consumidor, estar al tanto de sus opiniones, deseos y dudas.

*Vincular personas con el comercio electrónico: consideremos a las personas como ese "algo" que genera confianza, podemos hacer uso de aquellos cuyas opiniones son valoradas por la comunidad (Influencers) para que los consumidores asocien con ellos la compra por internet. Con esto se busca fomentar en el consumidor que la compra por internet no es del todo extraña y exclusiva, sino que cualquier persona puede llevarla a cabo. 
ESTRATEGIAS DE MARKETING PARA LA GENERACIÓN DE CONFIANZA EN LAS COMPRAS POR INTERNET

b) Asociar seguridad a través de una marca. El consumidor se siente más seguro al comprarle a un negocio establecido con una marca a hacerlo a un consumidor que vende a otros consumidores cualquier producto.

c) Asociar seguridad a través de sellos de garantía y acreditaciones: Para el consumidor es vital que el Portal muestre la información que acredite por instituciones dedicadas a este fin, que es seguro para realizar una transacción en internet, ya que esto simboliza al comprador que no será víctima de una estafa y disminuye la incertidumbre de riesgo.

\section{Las respuestas rápidas son lo más importante en la interacción vendedor-consumidor a través de Internet.}

En internet no existe la posibilidad de tener una interacción cara con el vendedor como ocurre en una compra tradicional. Sin embargo "el tiempo de respuesta" sustituye la importancia de esta variable. Que tan rápido se atiende una solicitud debe ser la prioridad.

\section{La interacción vendedor-consumidor debe ser a través de medios sociales.}

El consumidor actual se siente más seguro de interactuar a través de estas herramientas con un vendedor a distancia. Lo anterior, por la desconfianza de proporcionar su número telefónico existiendo otras posibilidades traídas por internet. La preferencia de los consumidores jóvenes por las Redes Sociales al correo electrónico, transforma el servicio al cliente para los negocios, convirtiéndolos en las herramientas principales para este fin.

\section{Instruir al consumidor en el proceso de compra por internet.}

Algunas personas no aceptan internet como canal de compra porque desconocen el proceso o lo consideran difícil, por lo cual optan por la compra tradicional. Como negocio, en el Portal es importante incluir un apartado donde se explique el proceso, paso a paso, de cómo se realizará la transacción.

\section{Fomentar los beneficios de la compra por internet.}

Estados Unidos percibió los beneficios del comercio electrónico y vio la posibilidad de globalizar el Black Friday, para que personas de todo el mundo accedieran a estos descuentos de tiendas norteamericanas, desarrollando el CyberMonday, elevando con esto las ventas a las nubes. En 
ESTRATEGIAS DE MARKETING PARA LA GENERACIÓN DE CONFIANZA EN LAS COMPRAS POR INTERNET

México ya existe una iniciativa a esta versión denominada "HotSale", donde empresas brindan descuentos exclusivos en sus tiendas digitales y se realiza del 30 de mayo al 2 de junio. Sin embargo, la difusión que se le da es poca, no solo se trata que los negocios participantes den a conocer esta información sino todos los medios en el país, esto permitirá incrementar el número de compradores online y acercar a la población que es renuente pero tienen un interés. Estas ofertas y promociones pueden disuadirlos a aceptar la transacción en línea.

\section{CONCLUSIONES}

Se identifica la existencia de factores cognitivos importantes que permiten que se genere en el consumidor una actitud de aceptación y de confianza hacia internet como canal de compra, más allá del factor tecnológico, el cual es mayormente asociado al hablar de seguridad en internet y que hace referencia únicamente a la tecnología empleada en el desarrollo del Portal (cifrado de datos, protocolos para la transmisión segura (HTTPS)). Estos son: transacción y privacidad, información/contenido, reputación, producto, asociación, beneficios y actitud hacia internet, cooperación e institucional. A través de la validación de estos factores por los jóvenes de la capital tabasqueña, se identifican estrategias de marketing, específicas a cada factor, a tomar a consideración para que en el estado crezca la aceptación e inmersión a la compra digital.

La decisión de utilizar una innovación cualquiera no es un acto instantáneo; más bien se trata de un proceso en el que se suceden decisiones y acciones por las que individuos y organizaciones pasan finalmente a la acción de utilizar o no esa innovación. Cuando el entorno de compra no es familiar, surgen más oportunidades de levantamiento de barreras que inhiben la adopción del nuevo entorno. En el caso de la utilización de un canal de compra los consumidores tienen que sobrepasar una serie de barreras, algunas cognitivas antes de realizar determinadas compras. Sugerir que el consumidor confiara solo por el factor tecnológico, es simplemente un error. La confianza es un elemento que se encuentra en la mente del consumidor y muchos desconocen los términos técnicos relacionados a informática y sistemas, por tanto pueden no comprender éste y requieren otros elementos de disuasión.

\section{RECOMENDACIONES}

- Dar más difusión a la compra por internet y a las campañas como CyberMonday y HotSale. El consumidor necesita escuchar que este no es un proceso difícil, cualquier persona puede realizarlo y ofrece muchos beneficios. 
ESTRATEGIAS DE MARKETING PARA LA GENERACIÓN DE CONFIANZA EN LAS COMPRAS POR INTERNET

- Dar a conocer las instituciones que regulan el comercio electrónico en el país y pueden apoyar al consumidor en caso de ser víctima de un fraude, ya que desconocen esta información y generará una percepción de valor, responsabilidad y confianza en el canal, sabiendo que al igual que en una compra tradicional, en el entorno digital también los protegen.

- Tener medios de pagos más bancarizados ya que en México existe una gran población que no cuenta con tarjeta de crédito o servicios de banca, por lo cual su acceso a la compra por internet es denegado. Los bancos deben aceptar más tarjetas de débito, porque quienes más compran en línea tienen entre 18 y 24 años y es más común que ellos usen débito. Uber es un negocio online de servicio de transporte privado que observó este detalle y lanzo su propia tarjeta de débito, este año, para atender a esta población. La tarjeta fue creada en alianza con la banca electrónica mexicana Bankaool y MasterCard, con esta los usuarios pueden pagar sus viajes y realizar compras, tanto en tiendas físicas como en Internet. Obtenerla es sencillo y se utiliza como una tarjeta de débito tradicional en la que la persona deposita dinero y puede disponer de este monto para gastar, como un "monedero electrónico". Sin duda se requiere más apoyo e innovación a este tipo de estrategias para un mayor crecimiento de la compra por internet en el futuro.

\section{REFERENCIAS BIBLIOGRÁFICAS}

Alzate, J. A. (2015). Factores que afectan la confianza de los consumidores antioqueños por las compras a través de canales de comercio electrónico. Medellín, Colombia.

AMIPCI. (2016). Estudio de comercio electronico. Obtenido de AMIPCI: https://www.amipci.org.mx/es/estudios

AMIPCl. (2016). Hábitos de los usuarios de internet en México. Obtenido de https://www.amipci.org.mx/es/estudios

Arango, L. F. (2013). Comercio electrónico, los riesgos que enfrenta América Latina para su masificación. Medellín.

Boen, D. (2004). Comercio Electrónico. México: Thomson.

CONDUSEF. (2016). Clonación de tarjeta: acciones para prevenir y cuidar tus finanzas. México. 
ESTRATEGIAS DE MARKETING PARA LA GENERACIÓN DE CONFIANZA EN LAS COMPRAS POR INTERNET

Egger, F. (2000). Trust me, I'm an online Vendor: Towars a model of trust for E-Commerce System Design.

García, P. L., \& Álvarez, F. J. (2000). El comercio electrónico y la seguridad de sus transacciones.

Kim, D., Song, Y., Braynov, S., \& Rao, R. (2001). A B-to-C Trust Model for On-line Exchange. Association for Information Systems.

Kotler, P., \& Armstrong, G. (2013). Fundamentos de Marketing. México: Pearson.

Martínez, F. J., Ortigueira, M., \& Pérez, M. (2006). Confianza del consumidor en la compra a través de internet: una propuesta de modelización basada en la jerarquía de aprendizaje estándar.

OECD. (1997). Committee for nformation, computer and communications policy. France.

Rojas, M. D., Arango, P., \& Gallego, J. P. (2009). Confianza para efectuar compras por internet.

Sampieri, R., Collado, C., \& Lucio, P. (2010). Metodología de la investigación (5ª ed.). México: McGrawHill. 